\title{
PRIMEIRA DESCRIÇÃO DO COMPORTAMENTO DE Bellactis ilkalyseae (CNIDARIA: ACTINIARIA) DURANTE O PROCESSO DE REPRODUÇÃO ASSEXUADA.
}

\author{
Thiago Gutemberg Lopes de OLIVEIRA \\ Paula Braga GOMES
}

G.P.A - Grupo de Pesquisa em Antozoários. Departamento de Zoologia, CCB, Universidade Federal de Pernambuco, Av. Professor Moraes Rego, 1235, Cidade Universitária CEP: 50670-901.

E-mail: gpa@ufpe.br

Recebido: $13 / 03 / 2005$

Aceito: 27/06/2005

\section{RESUMO}

A fissão longitudinal é um dos mecanismos mais comuns de reprodução assexuada em anêmonas-do-mar resultando em um rápido crescimento populacional. A espécie Bellactis ilkalyseae Dube, 1983, endêmica do Brasil, é extremamente gregária, porém nunca foi observado nem descrito o processo de reprodução assexuada da espécie. O presente trabalho descreve de forma preliminar o comportamento da espécie durante o processo de fissão longitudinal registrado em aquário. O material estudado foi coletado na praia de Guadalupe, litoral sul de Pernambuco, acondicionado em aquário com boas condições e alimentado diariamente com artémia. Um dos exemplares em observação realizou a reprodução por fissão longitudinal, num processo que durou três dias e que teve início pela base. O indivíduo originado começou a alimentar-se imediatamente após o processo e atingiu o tamanho do adulto cerca de três meses depois. O organismo parental permaneceu 10 dias sem alimentar-se e demorou 15 dias para a regeneração completa. Nos sete meses seguintes o indivíduo realizou 6 vezes o mesmo processo e nenhum dos organismos originados realizou reprodução assexuada.

Palavras chave: Aiptasiidae, fissão longitudinal, anêmonas-do-mar, Brasil.

\begin{abstract}
First description of the behavior of Bellactis ilkalyseae (Cnidaria: Actiniaria) during asexual reproduction

Longitudinal fission is one of the most common types of asexual reproduction in sea anemones, resulting in a quickly population increment. The species Bellactis ilkalyseae Dube, 1983, endemic from Brazil, has an aggregate distribution, nevertheless, it was never described the asexual process of reproduction on this species. The present work gives a preliminary description of the behavior of $B$. ilkalyseae during longitudinal fission observed in aquarium. Specimens collected in Guadalupe Beach, south of Pernambuco State (Brazil) were maintained in aquarium and fed with artemia. After four months a specimen started the longitudinal fission by the aboral end. All the process took three days and the new specimen started to feed immediately and reached the adult size in three months. The parental organism did not feed for ten days and took 15 days to complete
\end{abstract}

Tropical Oceanography, Recife, v. 33, n. 1, p. 67-72, 2005. 
regeneration. During the next seven months the same specimen made the fission process six times and no of the originated individuals made asexual reproduction.

Key words: Aiptasiidae, longitudinal fission, sea anemones, Brazil.

\section{INTRODUÇÃO}

Anêmonas-do-mar são animais polipóides sem esqueleto pertencentes à Ordem Actiniaria (GOMES, ZAMPONI \& SOLÉ-CAVA, 1998). Entre seus representantes há espécies dióicas e hermafroditas podendo ocorrer ainda reprodução assexuada (SHICK, 1991). A reprodução assexuada é utilizada como uma estratégia de crescimento populacional através do aumento da biomassa, apesar da diminuição do tamanho individual. Outra vantagem seria manter intacto o genótipo, já adequado ao meio (WILLIAMS, 1975) além de conseqüências ecológicas diretas como a redução da dissecação durante a emersão (OTTAWAY, 1973) e a oferta de refúgios para recrutas propiciados pelas agregações clonais (OTTAWAY, 1973).

Em anêmonas-do-mar a reprodução assexuada pode ocorrer através de quatro mecanismos diferentes: laceração pedal, fissão longitudinal, fissão transversal e alguns autores consideram ainda a gemação (FRANCIS, 1988). A laceração pedal e a fissão longitudinal são os mecanismos mais comuns entre os actiniários (MINASIAN, 1976).

No Brasil, entre as espécies que apresentam reprodução assexuada, Anthopleura variarmata Waltz, 1922, Telmatactis rufa (Verril, 1900) e Tricnidactis errans Pires, 1988 tiveram seus processos bem descritos. A primeira realiza fissão longitudinal ocorrendo pelas exoceles de forma simétrica gerando um indivíduo ligeiramente menor que o parental (BELÉM \& MONTEIRO, 1981). No caso de T. rufa o mecanismo utilizado é a fissão transversal (BELÉM \& SCHLENZ, 1989) e T. errans realiza laceração pedal (PIRES, 1988). Um estudo realizado com esta espécie em Mar del Plata, Argentina, revelou que o processo pode originar de 5 a 7 pequenos indivíduos que já se alimentam (EXCOFFON \& ZAMPONI, 1993). Outras espécies apresentam hábitos gregários e, devido a indícios como cicatrizes e grande irregularidade em sua morfologia, foram consideradas como possuindo a reprodução do tipo assexuada. Este é o caso de A. krebsi cuja estrutura clonal foi comprovada posteriormente com estudos genéticos (GOMES; ZAMPONI; SOLÉ-CAVA, 1998).

A espécie Bellactis ilkalyseae Dube, 1983 foi descrita inicialmente para a Bahia e já foi registrada na costa de Pernambuco (GOMES, 2002), Alagoas (PINTO, 2002), Arquipélago de Fernando de Noronha, Atol das Rocas, Arquipélago de Abrolhos e Espírito Santo (ZAMPONI, et al., 1998). Devido ao hábito gregário, a espécie é considerada como clonal, porém nunca foi descrito o seu comportamento reprodutivo assexuado. O presente trabalho descreve, de forma preliminar, o comportamento de Bellactis ilkalyseae durante o processo de fissão longitudinal registrado em aquário.

\section{MATERIAIS E MÉTODOS}

O material estudado foi coletado na praia de Guadalupe, litoral sul do estado de Pernambuco, em 18 de Setembro de 2001. Dois exemplares da espécie foram mantidos em aquário marinho provido de filtro (Skimmer), iluminação adequada, com fotoperíodo de 12 horas e boa circulação de água e ar. Os animais foram alimentados diariamente com artémia salina e pedaços de camarão até o final do experimento. As condições do aquário foram controladas através de testes químicos de salinidade, $\mathrm{pH}$, fosfatos e silicatos e as condições do mesmo mantidas constantes dentro dos padrões tradicionais para aquários marinhos. Os exemplares foram observados diariamente e seus movimentos anotados. Após o início do processo de fissão, as observações 
diárias foram realizadas em diversos horários e todo o comportamento das anêmonas era registrado. Durante o período de estudo os exemplares não foram movidos nem retirados do aquário e as condições foram mantidas constantes. Foram observados processos sucessivos de reprodução assexuada e as etapas dos mesmos foram registradas fotograficamente.

\section{RESULTADOS}

Um dos exemplares em observação foi um juvenil que atingiu o dobro do seu tamanho após quatro meses no aquário. No sexto mês o mesmo assumiu uma forma retraída, murcha, permanecendo assim por cerca de oito dias. Após este período o exemplar estendeu seu disco pedal mantendo-o estirado. $\mathrm{O}$ animal iniciou o processo de fissão pela base e demorou três dias para concluí-lo, desde o início do estiramento (Figura 1). O indivíduo originado consistia de um fragmento de coluna e disco oral ainda sem forma definida e bem transparente. O novo indivíduo já se alimentava logo após a fissão e em dois dias assumiu forma similar ao adulto, porém menor e ainda transparente. Cerca de 2 a 3 meses após a fissão o exemplar atingiu o tamanho do parental. Ao final do processo, o indivíduo parental apresentava o disco oral cerca de $1 / 4$ menor e iniciava a cicatrização da coluna. O indivíduo parental permaneceu fechado e murcho por cerca de dez dias após o processo, não se alimentando neste período. Só após uns 15 dias o animal estava completamente regenerado. Ambos indivíduos foram mantidos no aquário e desde a primeira fissão do parental, nos 7 meses seguintes, o mesmo realizou o processo 6 vezes. Nenhum dos 6 parentais originados realizou fissão, nem após atingir o tamanho do adulto.

\section{DISCUSSÃO}

A distribuição gregária em espécies de anêmonas-do-mar pode ser considerada como um indício de ocorrência de reprodução assexuada, principalmente se associada à presença de irregularidades morfológicas e de cicatrizes na coluna. No entanto, são conhecidas espécies que possuem hábitos gregários e não têm a capacidade de reprodução assexuada. Este é o caso da espécie brasileira Bunodosoma caissarum Corrêa em Belém, 1987. Por outro lado, a observação do processo de reprodução em aquário, se bem não determine a forma nem a freqüência de ocorrência do processo em campo, fornece a certeza da capacidade da espécie em realizar tal processo. Além disso, informações sobre o mecanismo assexuado permitem inferir sobre a possível importância do mesmo em populações naturais.

Entre a maioria dos representantes da Tribo Mesomyaria, a qual pertence a espécie, a forma predominante de reprodução é a assexuada, em especial por laceração pedal e/ou fissão longitudinal (SHICK, 1991). Ambos processos ocorrem, por exemplo, nas espécies Tricnidactis errans Pires, 1988 e Haliplanella lineata (Verrill, 1869), enquanto que a espécie Telmatactis rufa (Verrill, 1900) realiza fissão transversal e Carcinactis dolosa Riemann-Zürneck, 1975 parece não utilizar o processo assexuado para reproduzir-se (PINTO, 2002).

Em B. ilkalyseae foi observada apenas a fissão transversal, não havendo indícios de outro tipo de reprodução assexuada na espécie. De fato, entre as agregações encontradas em poças e fendas no mediolitoral inferior e infralitoral de Pernambuco, não foram encontrados indivíduos de tamanho reduzido, como os fragmentos originados através da laceração pedal (GOMES, Obs. Pess.). Também não foi observado o processo em aquário, mesmo após dez meses de observações. A outra espécie da família Aiptasiidae presente no Brasil, Aiptasia pallida Verrill, 1864, realiza o processo de laceração pedal como principal forma de reprodução.

Tropical Oceanography, Recife, v. 33, n. 1, p. 67-72, 2005. 
Pelo presente estudo, a espécie $B$. ilkalyseae parece realizar um processo similar à $A$. varioarmata Watzl, 1922, já que em ambas o processo inicia-se pela base e origina indivíduos de tamanhos diferentes (BELÉM \& MONTEIRO, 1981). No entanto, a existência de poucos estudos que descrevam os processos de reprodução assexuados dificulta a comparação. A elevada freqüência de reprodução observada em aquário demonstra a grande capacidade de divisão e regeneração da espécie. Apenas estudos em campo poderão determinar a contribuição deste processo para a população.

Em Pernambuco B. ilkalyseae já foi encontrada nas praias do Pina, Porto de Galinhas, Serrambi, Guadalupe e Tamandaré. Em todos os locais forma agregações no mediolitoral em locais sombreados, com aporte de água e, às vezes se estende ao infralitoral superior. Não foram registrados indivíduos isolados, indicando que a reprodução assexuada deve ser predominante. Em populações intermareais a reprodução assexuada está, geralmente, associada à proliferação clonal espacialmente restrita, levando a populações altamente estruturadas (BILLINGHAM \& AYRE, 1997). Considerando o estresse mais elevado que caracteriza a zona entremarés (BARNES, FINLAYSON \& PIATIGORSKY, 1963), a reprodução assexuada pode ser vantajosa por produzir genótipos já adaptados ao ambiente, além de formar agregações que auxiliam na proteção contra a dessecação. Além disso, muitas vezes o rápido crescimento populacional auxilia na colonização de novos habitats, o que caracteriza, por exemplo, espécies invasoras. No caso de B. ilkalyseae, a espécie é, até o presente, endêmica do Brasil, distribuindo-se no nordeste do país e no estado do Espírito Santo. A ausência de registro da espécie em outras localidades bem estudadas como os litorais de São Paulo e Rio de Janeiro, leva a crer que a mesma possa apresentar alguns requerimentos ambientais específicos que condicionem sua distribuição. Outra possibilidade a ser considerada é que a predominância da proliferação clonal mantenha as populações restritas, diminuindo sua capacidade de dispersão e colonização de novos ambientes. Não há ainda estudos sobre a biologia reprodutiva da espécie, necessários para esclarecer a questão. 


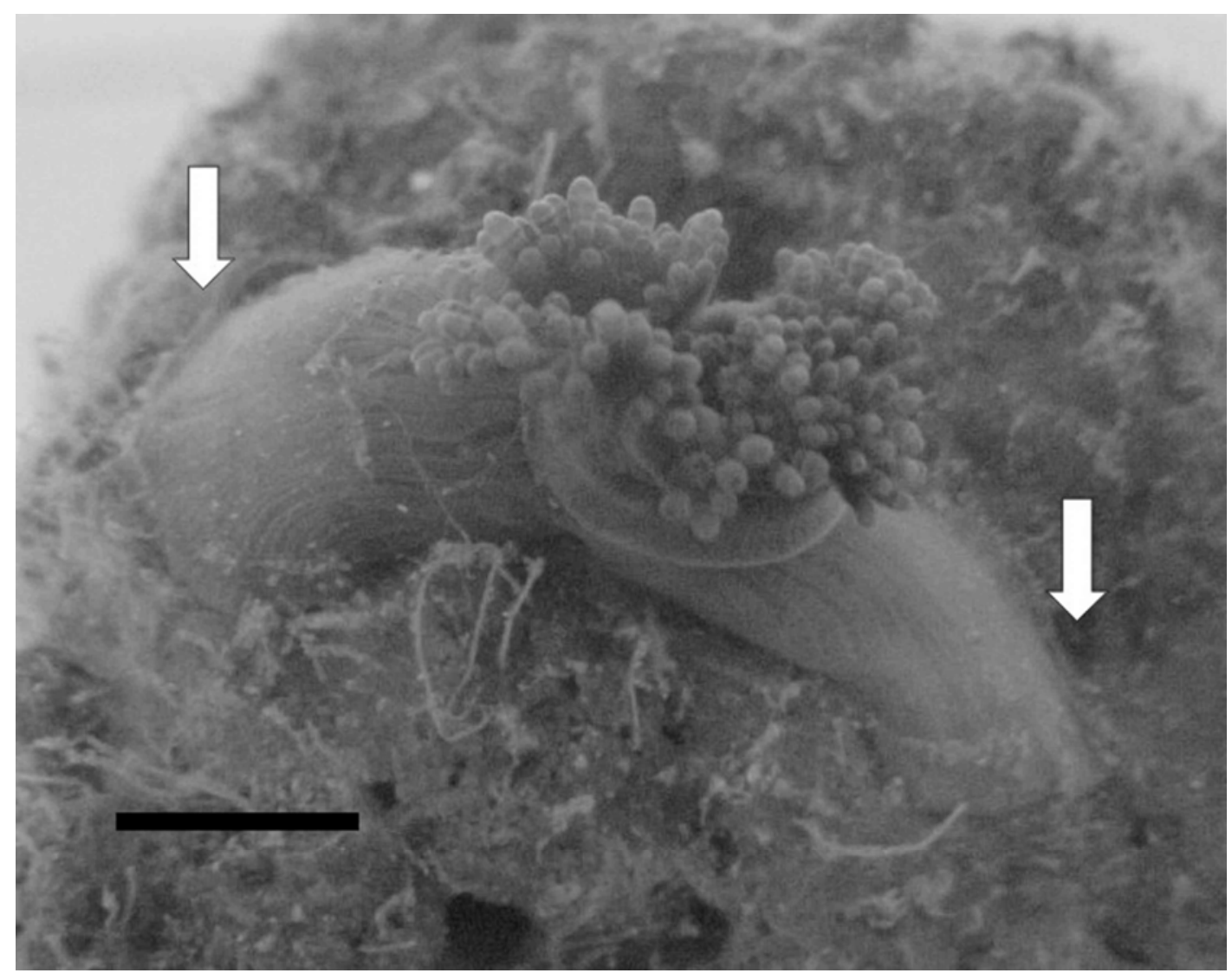

Figura 1. Bellactis ylkaliaseae durante processo de fissão longitudinal em aquário. As setas indicam o disco pedal estirado em início de divisão. A escala equivale a $1,5 \mathrm{~cm}$.

\section{REFERÊNCIAS BIBLIOGRÁFICAS}

BARNES, H.; FINLAYSON, D.M.; PIATIGORSKY, J. The effect of desiccation and anaerobic conditions on the behaviour, survival and general metabolism of three cooomn cirripedes. J. Anim. Ecol., Oxford, v. 32, p. 233-252, 1963.

BELÉM, M.J.C.; MONTEIRO, D.C. Fauna de Cnidários do Rio de Janeiro. III. Anthopleura varioarmata Watzl, 1922 (Actiniaria, Endomyaria), uma nova ocorrência de Actiniidae.

Seminários de Biologia Marinha - Acad. Bras. Ci., Rio de Janeiro, p.193-203, 1981.

BELÉM, M.J.C.; SCHLENZ, E. First records of an Isophellidae (Cnidaria, Actiniaria) in Brazil, with the redescription of Telmatactis rufa (Verrill, 1900) and observations on its assexual reproduction. An. Acad. Bras. Ci., Rio de Janeiro, v. 61, n.3, p. 343-353, 1989.

Tropical Oceanography, Recife, v. 33, n. 1, p. 67-72, 2005. 
BILLINGHAM. M.R.; AYRE, D. J.. Asexual reproduction and genetic determination of colour patterns within populations of the subtidal sea anemone Anthothoe albocincta. Mar. Ecol. Prog. Ser. Oldendorf/Luhe, v. 156, p. 121-130, 1997.

EXCOFFON, A.; ZAMPONI, M. Anémonas de Mar del Plata y localidades vecinas. IV.

Tricnidactis errans Pires, 1988 (Actiniaria, Haliplanellidae) Iheringia, Ser. Zool., Porto Alegre, v. 75, p. 47-53, 1993.

FRANCIS, L. Cloning and aggression among sea anemones (Coelenterata: Actiniaria) of the rocky shore. Biol. Bull., Woods Hole, v. 174, p. 241-253, 1988.

GOMES, P.B. Anêmonas-do-mar (Cnidaria, Actiniaria) de Pernambuco (Brasil). Em: Diagnóstico da Biodiversidade de Pernambuco. M. Tabarelli; J.M.C. Silva (Coord). SECTMA e Editora Massangana, Recife, 2002, v. II, p. 343-364.

GOMES, P.B., ZAMPONI, M.O., SOLÉ-CAVA, A.M. Asexual reproduction and molecular systematics of the sea anemone Anthopleura krebsi Duchassaing \& Michelotti, 1860 (Actiniaria: Actiniidae). Rev. Biol. Trop. Costa Rica, v.51, n.1, p.147-154, 2003.

MINASIAN, L.L. Characteristics of asexual reproduction in the sea anemone Haliplanella luciae reared in the laboratory. In: Coelenterate ecology and behaviour. Mackie Plenum Press. New York, 289-298. 1976

OTTAWAY, J.R. Some effects of temperature, desiccation and light on the intertidal anemone Actinia tenebrosa Farquhar (Cnidaria: Anthozoa). Aust. J. Mar. Fresh. Res., v.24, p, 103-126, 1976.

PINTO, S.M. Anêmonas-do-mar com acôncios (Anthozoa: Actiniaria, Mesomyaria): analyses morfológica e molecular. Tese de Doutorado, Universidade de São Paulo, São Paulo, 2002, 186p.

PIRES, D.O. Tricnidactis errans n.gen n.sp. (Cnidaria, Actiniaria, Haliplanellidae), from Guanabara Bay, Rio de Janeiro, Brazil. Rev. Brasil. Biol, Rio de Janeiro, v. 48, n. 3, p. 507-516, 1988.

SHICK, J. M. A functional biology of sea anemones. London: Chapman \& Hall, 1991. 395 p.

WILLIAMS, R.B. Catch-tentacles in sea anemones:occurrence in Haliplanella luciae (Verrill) and a review of current knowledge. J. Nat. Hist., Londres, v.9, p. 241-248, 1975

ZAMPONI, M. O.; BELEM, M.J.C.; SCHLENZ; E.; ACUÑA, F. Occurrence and some ecological aspects of Corallimorpharia and Actiniaria from shallow waters of the south American Atlantic Coasts. Physis, Sec. A, Buenos Aires, v. 55, n. 128-129, p. 31-45, 1998. 